\title{
Intra-operative neurophysiological monitoring
}

\author{
Zulfiqar Ali, Parmod K. Bithal
}

\begin{abstract}
Intraoperative neurophysiological monitoring has achieved importance due to complexity of cranio-spinal surgical procedures being performed frequently these days. Many studies have proven a decreased neurological complication rate after its introduction. It is broadly of two types: Sensory evoked potentials and motor evoked potentials which are further sub-divided. Its use during surgery requires a controlled anaesthesia technique with no or minimal influence on its recording. Its success depends upon three way communication among the surgeon the neurophysiologist and the anaesthesiologist.
\end{abstract}

Key words: Evoked potentials, intra-operative, neurophysiological monitoring

\section{INTRODUCTION}

The nervous system has a unique property of exchanging information from one point to another point by the generation of electrical activity. Monitoring of this electrical activity helps in the assessment of the functional status of the nervous system. Intra-operative neurophysiologic monitoring can help in the early detection of neuronal ischaemia due to the unfavourable physiological environment or surgical insult. Appropriate manoeuvres if taken timely may help to reduce the neurological ischaemia and reduce the operative morbidity. Correctable factors at surgery include hypotension, hypoxia and excessive compression from surgical retractors, pressure

Department of Anaesthesiology and Critical Care, Division of Neuroanesthesiology, Sheri Kashmir Institute of Medical Sciences, Srinagar, Jammu and Kashmir and Department of Neuroanaesthesiology and Critical Care, Neurosciences Center, All India Institute of Medical Sciences, New Delhi, India

Address for correspondence:

Dr. Parmod K. Bithal, Department of Neuroanaesthesiology and Critical Care, Neurosciences Center, $7^{\text {th }}$ Floor, All India Institute of Medical Sciences, New Delhi - 110 029, India.

E-mail: bithal.parmod@gmail.com

\begin{tabular}{|l|l|}
\hline \multicolumn{2}{|c|}{ Access this article online } \\
\hline Quick Response Code: & Website: \\
\cline { 1 - 2 } & www.jnaccjournal.org \\
\cline { 1 - 2 } & \\
\hline & \\
\hline
\end{tabular}

from bony structures or haematomas or mechanical stretching.

\section{BASICS OF EVOKED POTENTIALS}

Evoked potentials record the electrical potentials produced after stimulation of specific neural tracts. An evoked potential is plotted as a record of voltage versus time. The initial period during which a neuronal tract gets stimulated is represented as an 'initial artefact'. This is followed by an actual stimulation of the neuronal tract indicated by the 'neuronal response'. The neuronal response is recorded as a series of peaks and valleys [Figure 1]. Peaks may be positive or negative (with respect to the active electrode) and are accordingly plotted as downwards or upwards. The amplitude is defined as the distance from the peak to adjacent trough. The time from the stimulation to the peak is called as latency [Figure 1]. Peaks are usually named by convention - I through $\mathrm{V}$ or as $\mathrm{Pa}, \mathrm{Pb}$, etc. They may be named by polarity and latency - P standing for positive and $\mathrm{N}$ standing for negative, followed by the latency in milliseconds (msec) (e.g. N20).

To generate sensory evoked potentials (SEP), a monophasic stimulation for a period of $100-300 \mu \mathrm{s}$

This is an open access article distributed under the terms of the Creative Commons Attribution-NonCommercial-ShareAlike 3.0 License, which allows others to remix, tweak, and build upon the work non-commercially, as long as the author is credited and the new creations are licensed under the identical terms.

For reprints contact: reprints@medknow.com

How to cite this article: Ali Z, Bithal PK. Intra-operative neurophysiological monitoring. J Neuroanaesthesiol Crit Care 2015;2:179-92. 


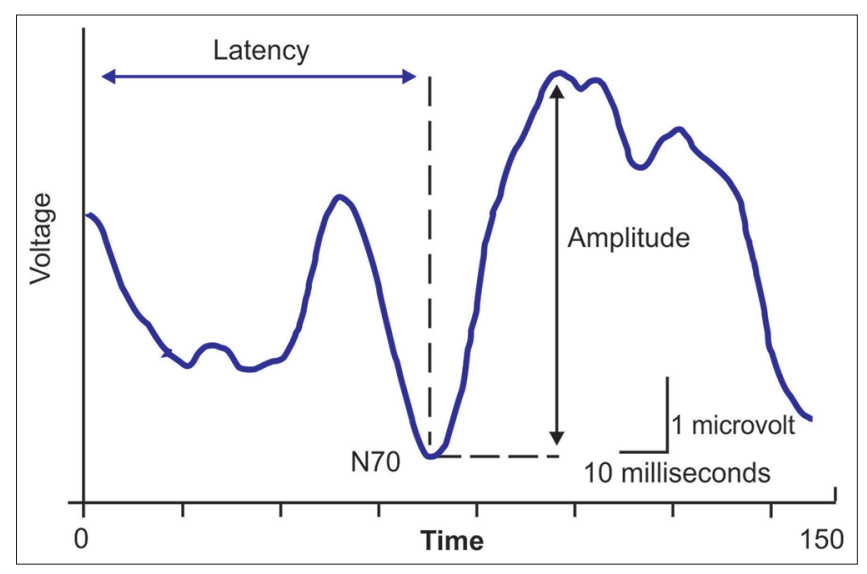

Figure 1: Evoked response tracing of amplitude versus time after the stimulus. The measurement of latency and amplitude for the negative peak at $70 \mathrm{~ms}$ (N70) is shown

is given. A rapid stimulation rate should be avoided because they degrade the SEP waveform. In clinical setting, a rate of 3-6 stimuli per second is used. As a general principle, an amplitude reduction of $50 \%$ or latency increase of $10 \%$ of SEP is considered as significant change, although smaller changes may indicate impending neurological ischaemia.

\section{CLASSIFICATION}

The evoked potentials may be sensory (somatosensory evoked potentials [SSEPs], brainstem auditory evoked potentials [BAEPs] and visual evoked potentials [VEPs]) and motor evoked potentials (MEPs).

\section{SOMATOSENSORY EVOKED POTENTIALS}

The SSEP is the most commonly monitored SEP. The peripheral sensory nerves are stimulated electrically. The elicited response is measured along the sensory pathway. The large nerves (mixed motor and sensory nerves along with their spinal roots) mainly used in clinical practice are median (C6-T1), ulnar (C8-T1), common peroneal (L4-S1) and posterior tibial (L4-S2) nerves. During electrical stimulation, there is activation of the large-diameter, fast conducting Ia muscle afferents and group II cutaneous nerve fibres (1). As a result of neural stimulation, there is both an orthodromic (propagating in the normal direction) and antidromic (propagating in the reverse direction) neural transmission. The orthodromic motor stimulation elicits a muscle response, which is seen as a foot or hand twitch, and the orthodromic sensory stimulation produces the SSEP.

The responses from the upper limb are measured from electrodes placed over the antecubital fossa, supraclavicular fossa, cervical spine and cortex; and for the lower extremity, they are recorded from the popliteal fossa, along the spinal cord (surface or epidural electrodes) and at cervical and cortical locations. Response recordings are usually obtained from multiple recording sites to verify that the nervous system is stimulated and to identify the location of neural compromise if the response is lost.

The cortical response is best recorded over the primary somatosensory cortex appropriate for the nerve, which is stimulated. The major cortical peaks recorded after median nerve and posterior tibial nerve stimulation (N20 and P38, respectively) are likely the result of the thalamocortical projections to the primary sensory cortex.$^{[1]}$ Responses recorded posteriorly over the cervical spine probably represent responses of the tracts in the spinal cord or brainstem. ${ }^{[1]}$

\section{Somatosensory evoked potential as an indicator of ischaemia from reduced cerebral perfusion}

The interval between the P14 and the N20 peaks of the SSEP is the central conduction time (CCT). It is used as an indicator of the ischaemic changes in the central somatosensory nervous system structures. A prolongation of the CCT is taken as an indication of the beginning of ischaemia. ${ }^{[2]}$

Animal experimentations by Branston et al. ${ }^{[3]}$ have shown that there is a direct relationship between the time it takes for the SSEP to disappear and the degree of ischaemia. Experiments in baboons showed that the SSEP disappears when cerebral blood flow falls below $15-18 \mathrm{~mL} / 100 \mathrm{~g} / \mathrm{min}$, but a more severe decrease (to about $10 \mathrm{~mL} / 100 \mathrm{~g} / \mathrm{min}$ ) in blood flow is necessary to disturb ionic homeostasis causing permanent neuronal damage ${ }^{[4]}$ Studies in humans by Symon et al. ${ }^{[5]}$ in aneurysm surgeries have shown that there is a relationship between the time taken for the N20 peak of the SSEP to disappear after occlusion of an artery and the risk of occurrence of permanent neurological deficit. The time it takes for the SSEP to no longer be detectable following occlusion (clamping) of a branch of the middle cerebral artery (MCA) was found to be crucial to the neurological outcome. The shorter the time it takes, the higher the risk of the permanent neurological deficit. If the time is $<2 \mathrm{~min}$, the risk is high for the permanent deficit. Occlusion causes a lesser degree of ischaemia when it takes a longer time for the SSEP to disappear. In patients in whom it took 4 min or more for the SSEP to disappear, 20 min of the absence of the N20 peak of the SSEP was tolerated when the carotid artery or the MCA was occluded. If the time taken for the N20 peak to disappear is $<4 \mathrm{~min}$, the estimated time of tolerance is reduced to $10 \mathrm{~min} \cdot{ }^{[6]}$

Because the SSEP from the upper limbs is generated in the cerebral cortex supplied by the MCA, it is used intra-operatively to detect ischaemic changes during 
temporary clipping for aneurysms of the internal carotid and middle cerebral arteries. Symon and Murota ${ }^{[7]}$ suggested that the use of SSEP elicited from the lower limbs (posterior tibial nerve stimulation) is more effective in detecting ischaemia caused by occlusion of the anterior cerebral artery than the use of SSEP elicited from the median nerve.

In clinical practice, the ability of SSEPs along with MEPs to help in the detection of motor deficits due to subcortical ischaemia has been found to be variable. ${ }^{[8]}$

\section{Somatosensory evoked potential as an indicator of brainstem manipulation}

The value of intra-operative monitoring of SSEP in patients undergoing posterior fossa surgeries where there may be brainstem manipulation is not as helpful as monitoring of auditory brainstem response, because there are no brainstem relay nuclei in the somatosensory system. The fibre tract of the medial lemniscus that passes through the brainstem might be affected by brainstem manipulation. However, the recorded changes in the cortical SSEP are not significant enough to help in the detection of neuronal ischaemic changes.

\section{Use of somatosensory evoked potential} monitoring for identification of sensory cortex Monitoring of evoked potentials using the cortical component (N20) of the median nerve SSEP with use of bipolar recording strips placed on the cortex helps in the identification of the central gyrus separating the motor and sensory strips. A phase reversal (initial wave changes from positive to negative) at the central gyrus as a result of the horizontal nature of the dipole generator located in the gyrus helps identify the sensory cortex.

\section{Somatosensory evoked potential monitoring in spinal surgery}

Intra-operative monitoring of spinal cord function is indicated in those surgeries where the blood supply to the spinal cord could be compromised or the surgical procedures where there is manipulation of the spinal cord. This may be seen in excision of spinal cord tumours, corrective surgery for scoliosis, spinal stenosis and spinal cord injuries.

Intra-operative monitoring of SSEPs only monitors the sensory pathways of the spinal cord. The non-sensory pathways such as the descending motor pathways may be injured without any noticeable change occurring in the recorded SSEP. This is mainly because of the fact that the SSEP is transmitted predominantly through the posterior columns with blood supply from the posterior spinal artery. The motor tracts are anteriorly located with their blood being supplied by the anterior spinal arteries. There are few limitations to this concept regarding the separation of the motor and sensory tracts of the spinal cord that should be kept in mind in clinical practice. First, the ischaemic injury may not always exactly respect the division between the ventral and dorsal cord, so that vascular injuries to the ventral portion of the spinal cord can be reflected in changes in the SSEP. ${ }^{[9]}$ Second, mechanical injury to the spinal cord may affect both the ventral and dorsal portions of the spinal cord. Third, the pathways contributing to the SSEP are not purely limited to the dorsal column system $^{[9]}$ and pathways in the lateral cord such as the dorsal spinocerebellar tract might contribute to the conduction of the SSEP. Fourth, insults to the ventral portion of the spinal cord might cause a 'spinal shock,' and thereby, affect the SSEP transiently. This might be because of the abundant connections in the spinal cord that connect different parts of the spinal cord. Therefore, the ability of the SSEP to predict the most motor deficits probably results from insults that affect the entire spinal cord.

\section{RECORDING}

To record the SSEP in the operating room, several recording channels are used to differentiate between the different components of elicited response. Most of the current machines used in the operating room have 16 amplifiers and can record up to 16 channels simultaneously.

The cortical (N20) and midbrain (N18) potentials evoked during stimulation of the upper limb SSEP are recorded with the active electrode placed over the contralateral parietal cortex $-2 \mathrm{~cm}$ behind C3 or C4 called C3' or C4' (10-20 system) $)^{[10]}$. The reference electrodes for such recordings are often placed on the forehead. Using an active electrode on the scalp and a non-cephalic reference electrode placed on the (shoulder or sternum) helps in a better recording of early subcortical components of the SSEP in response to median nerve stimulation (P9, P11 and P14-P16). ${ }^{[1]}$

Recordings of the lower limb SSEPs are usually done with the active electrode placed on $\mathrm{Cz}$ (or $2 \mathrm{~cm}$ behind) and the reference electrode either at a frontal scalp position or at a non-cephalic location (shoulder or upper neck). The most commonly monitored spinal/brainstem potentials are the P9, P11 and P14-P16 and N18. The P9 is generated where the nerves from the brachial plexus enter the spine; the P11 is generated internally in the dorsal horn of the spinal cord. The P14-P16 is generated close to or in the dorsal column nuclei.

There are cortical components (P40 or P37) and subcortical components (N34 and N21) of the lower limb SSEP that can have value for intra-operative monitoring. Recordings of the P40 or P37 components of the cortical 
components of the lower limb SSEP are usually made with the active electrode placed $2 \mathrm{~cm}$ posterior to the vertex $\left(\mathrm{Cz}^{\prime}\right)$ and the reference electrode placed on the forehead. The N34 component of the subcortical responses originates in the brainstem. It is typically recorded using an Fpz to a cervical electrode.

It is readily recorded in most patients but can be of low amplitude. The advantage of monitoring this potential during spine surgery is that it is much less sensitive to anaesthetic effects than the cortical potentials. The N21 component of the SSEP is elicited by lower limb stimulation and recorded from an electrode placed at T12 vertebra with the reference electrode on the iliac crest.

\section{Limitations of somatosensory evoked potentials}

A critical limitation of SSEP monitoring is that the value is based on the calculated averages of stimuli which may take several minutes to change following an acute insult. In 2004, Hilibrand et al. ${ }^{[12]}$ compared SSEP and MEPs and found that SSEPs lagged behind MEP changes by an average of $16 \mathrm{~min}$. Furthermore, SSEPs have a low sensitivity for detection of nerve root injury, and thus, may miss injury resulting from misplaced pedicle screw or nerve root traction.

These deficiencies limit the overall efficacy of SSEPs as a standalone monitoring technique; and thus, should be used mainly as an adjunct to other forms of monitoring.

\section{Brainstem auditory evoked potentials}

The eighth cranial nerve (CN VIII) is at risk of being injured by surgical procedures in microvascular decompression to relieve trigeminal neuralgia, hemifacial spasm and glossopharyngeal neuralgia. The preservation of auditory function with a better functional outcome in terms of hearing preservation and less incidence of facial nerve paresis is now possible during the removal of vestibular schwannoma because of advancements in operative techniques and better intra-operative neurophysiological monitoring of the auditory nerve. ${ }^{[13]}$

In BAEPs monitoring, the auditory stimulator discharges acoustic clicks to the operated ear through an earphone, while the scalp and earlobe electrodes record the evoked potential. ${ }^{[14]}$

Before starting the surgical procedure, the stimulus (intensity in decibels) is adjusted until the patient hears the sound of the click. During the surgical procedure, this is followed by delivering the stimulus at several decibels higher than the measured threshold. ${ }^{[14]}$ White noise is applied at lower decibels to the contralateral ear. The peaks of the evoked electrical potentials are classified as Wave I through Wave V.

Wave I is produced by the extra-cranial portion of $\mathrm{CN}$ VIII, wave III by acoustic relay nuclei and tracts deep in the midline of the lower pons, and wave $\mathrm{V}$ by the lateral lemniscus and inferior colliculus in the contralateral pons. When considering BAEP waveform shifts, Waves I, III and V carry the most clinical significance. ${ }^{[15]}$

Increased peak latencies of Waves I, III, and $\mathrm{V}^{[16]}$ high inter-aural latency differences, ${ }^{[14]}$ decreased amplitudes of Waves I and $\mathrm{V},{ }^{[14]}$ and increased inter-peak latencies between Waves I-III, III-V, and I- $\mathrm{V}^{[14]}$ are regarded as the main clinically significant neurophysiological changes. Inter-peak latencies are not susceptible to influences from external factors such as age and are more useful clinically. ${ }^{[17]}$

The prognostic power of BAEPs to detect neuronal damage is mainly based on the preservation of the waveforms. ${ }^{[18]}$ When actual changes occur during monitoring of BAEPs, the presence or the degree of severity of post-operative deficits cannot be accurately predicted. ${ }^{[19]}$ However, the occurrence of these changes in the BAEP waveform alerts the surgeon to the potential nerve damage. Appropriate steps are taken by alteration of the surgical procedure.

\section{Limitations of brainstem auditory evoked potentials}

The use of BAEPs comes with several limitations.

As the stimulus response is summed and averaged, there is a significant time delay up to from seconds to several minutes. ${ }^{[19]}$ The data obtained are not dynamic, as BAEPs data obtained are the data that were applicable several seconds or minutes before. ${ }^{[19]}$

The second major limitation is that the waveform shifts are not only caused by trauma, similar changes may be caused by anaesthesia, hypothermia and irrigation of cold saline. This may cause difficulty in the interpretation of the data obtained and influence the decision making during surgical dissection. ${ }^{[15]}$

\section{Electrocochleography and direct compound nerve action potentials}

Electrocochleography (ECOG) and direct compound nerve action potential (CNAPs) are 'near-field' techniques as the stimulation evokes and records an electrical response, which is near its origin on the auditory nerve. This is in contrast to BAEP, which is a 'far-field' technique as the auditory response is measured on the scalp.

As the evoked potential is recorded from the nerve itself, the intra-operative neuromonitoring (IONM) bypasses the noise and artefact created in far-field IONM. There is 
a reduction in the number of stimuli averages required for better amplitude leading to a faster and a better assessment of the nerve function. ${ }^{[20]}$

Both ECOG and direct CNAPs use electrodes to measure potentials generated from the auditory nerve, with some small differences. In ECOG, the stimulating electrode administers click impulses, similar to BAEP multiple responses are averaged for a distinct wave pattern to emerge. ${ }^{[15]}$ For ECOG, electrodes are placed trans-tympanically on the middle ear promontory of the pathological ear with reference and ground electrodes on the ipsilateral earlobe and on the forehead, respectively. ${ }^{[15]}$

In direct CNAPs, the action potential is directly measured from the acoustic nerve itself. ${ }^{[15,20]}$ The recording electrode is placed directly on the acoustic nerve, the negative electrode is attached to the mastoid of the contralateral ear and a reference electrode is placed on the scalp. ${ }^{[15,20]}$ Intra-operatively the recording electrode may be placed proximal to the tumour being resected, ${ }^{[15,20]}$ with Gelfoam between the electrode and nerve to reinforcing the contact. Such as the BAEP and ECOG, a click stimulus is applied through an earphone and the resulting compound nerve action potential is measured.

The CNAPs are mainly seen as negative peaks with high amplitudes. ECOGs have 2 action potential peaks designated ' $\mathrm{N} 1$ ' and ' $\mathrm{N} 2$ '. The absolute loss of $\mathrm{N} 1$ on $\mathrm{ECOG}^{[2,21]}$ or on direct recording is frequently associated with post-operative hearing deficiency. Changes in the latency or amplitude of N1 on either ECOG or direct recording are also electrophysiological signs suggestive of injury. ${ }^{[20,21]}$

\section{Intra-operative monitoring of the facial nerve cranial nerve VII}

Post-operative facial nerve weakness increases the post-operative morbidity as a result of the inability to blink, secrete tears and improper articulation.

The House-Brackmann Grading Scale, which grades the facial nerve weakness from Grade I through Grade VI has shown better outcomes as a result of advances in micro-neurosurgery and intra-operative neuromonitoring of the facial nerve. ${ }^{[22]}$

\section{Electromyography}

They can be spontaneous or free running electromyography (EMG) or triggered EMG. Free run EMGs can be continuously monitored when peripheral nerve or roots are at risks of potential injury. They are useful in facial or other $\mathrm{CN}$ monitoring, the release of tethered cord and selective dorsal rhizotomy. Free-run EMGs are the most useful for procedures at a spinal level where nerve roots are most likely to be at risk of injury.
Most commonly, this involves procedures at the lumbar level, but cervical level procedures are also amenable to free-run EMG monitoring.

No stimulation is needed to generate them and continuous recording is performed from preselected muscle groups depending upon nerve root at risk. One muscle group per root is generally considered as sufficient, but due to high vulnerability of C-5 to damage during cervical spine surgery, many surgeons monitor two muscle groups at this level, namely deltoid and biceps. ${ }^{[23,24]}$

A healthy, normal nerve root does not show any muscle activity, that is, either a flat line or silence if audio feedback is used. Irritation of the nerve root due to traction or thermal injury will lead to spikes or burst of activity termed as neurotonic discharges. When the intensity of injury increases, amplitude and frequency of these discharges will increase and trains of activity may be observed. ${ }^{[23,25]}$

During its monitoring muscle relaxant has to be avoided. Unlike Tc MEPs, spontaneous EMG allows continuous feedback throughout the procedure.

Free-run EMGs are sensitive to temperature changes and frequently causes of false positive, spontaneous EMG activation are irrigation with cold saline and use of diathermy. Various studies have shown it to possess high sensitivity but low specificity. ${ }^{[23,26]}$

\section{Triggered electromyography}

It is used to assess the accuracy of pedicle screw placement. It is based on the principle that intact cortical bone should insulate a properly placed pedicle screw from the adjacent nerve root. However, if there is medial pedicle breech, the pedicle screw would be relatively poorly insulated. Thus, by electrically stimulating the pedicle screw directly and electromyographically assessing the lowest threshold voltage at which compound muscle action potentials (CMAPs) are generated, one can assess the likelihood of medial pedicle breach. ${ }^{[27]}$

Triggered EMGs are particularly useful in minimally invasive spine surgery, because of limited visualisation of the anatomical landmark. In such situation stimulation of pedicle taps and K-wires may be used to evaluate for accurate screw trajectory. Notably, in the setting of pre-operative nerve root deficit, nerve conduction may be impaired, requiring higher thresholds for stimulation. Nerve conduction can be assessed intra-operatively by direct stimulation of the nerve root at low voltage and assessing for generation of CMAPs. ${ }^{[27]}$

There is no consensus yet on warning criteria for pedicle perforation. However, Raynor et al..$^{[28]}$ in 2007 reported 
results of more than 4800 consecutive lumbar pedicle screw placement with triggered EMG results when compared with post-operative computed tomography scan in each case. They reported that with a threshold of more than $8.0 \mathrm{~mA}$, there was a $99.5-99.8 \%$ likelihood of intra-osseous screw placement (95\% confidence interval), but the high false positive rate at this threshold may lead to unnecessary delays in surgical time and revision of adequately placed screws. More recently Parker et al. ${ }^{[29]}$ reported results of 2450 consecutive lumbar screw placements and found that by using a threshold cut-off of $<5 \mathrm{~mA}$, they were able to maintain an acceptability sensitivity of $43.4 \%$ for medial screw breach, while limiting the false positive rate.

Very few studies have reported the usefulness of triggered EMGs for thoracic pedicle screw placement according to Raynor et al. ${ }^{[30]}$ for upper thoracic (T2-T6) pedicle screw insertion, electrodes are placed at corresponding intercostals space at the nipple line and CMAP activity from intercostals muscles is assessed. For lower thoracic (T7-T12) screws, paired electrodes are placed along the nipple line at evenly separated distance between the lower margin of the $10^{\text {th }}$ rib and iliac ridge. ${ }^{[31]}$ In most instances of lower thoracic screw monitoring, CMAP activity from the rectus abdominus muscles is assessed. ${ }^{[30]}$ The same authors ${ }^{[30]}$ observed that above a threshold of $6.0 \mathrm{~mA}, 100 \%$ of screws were intra-osseous. For screws with a threshold of $<6.0 \mathrm{~mA}$, $28.5 \%$ of screws were found to be medial breaches. Thus, the authors recommended using a threshold of $<6.0 \mathrm{~mA}$ as a warning criterion for likely pedicle perforation.

EMG monitoring of pedicle screws is more reliable in the thoracolumbar junction and lumbar spine. However, it acts as additional tools to minimise serious injury in deformity surgery. Although insufficient when used alone as a safety measure, it provides additional information that can help surgeon and lower risk to the patient.

\section{Limitations of triggered electromyography}

The chief limitation of triggered EMG is the high false positive rate. Common causes of this problem are multiple passes, within the same pedicle leading to diminished pedicle integration and a wet operative field which may result in direct current conduction to the adjacent nerve root. ${ }^{[27]}$ One must note that the most studies on triggered EMG simply state the incidence of pedicle perforation, and there is very little data on clinical correlation with these findings. However, this monitoring modality is a valuable technique for increasing the safety of spine surgery.

\section{Electromyography of facial nerve}

The device for facial nerve monitoring has a stimulator probe for stimulation and a 'sensor' probe to detect the contractions of the facial muscles. A minimum of 2 channels is used to monitor the orbicularis oris and orbicularis oculi muscles.

A pair of needle electrodes is placed in the orbicularis oris and orbicularis oculi muscles while another is placed on the forehead or shoulder for grounding. The baseline motor unit potential (MUP) is recorded for future comparisons. The stimulator probe acts as a navigator to locate the facial nerve. The ideal location to determine the facial nerve is near the brainstem as it is proximal to the area of resection. ${ }^{[32]}$

On applying the electrical stimulus which exceeds the threshold of the patient's facial nerve, an action potential is generated which causes twitching of the facial muscles. The sensor detects these facial movements. The mechanical energy emits a sound alarm providing a direct, immediate and real-time feedback. ${ }^{[33]}$ Recently, the facial muscle MUPs have been projected onto an oscilloscope for better visualisation.

There are various types of MUPs observed on intraoperative EMG study.

'Spike' is defined as a single MUP while as 'burst' is a short chain of MUPs [Figure 2]. When there is a sustained streak of MUPs it is called as a 'train'. Train MUPs with a frequency of $>30 \mathrm{~Hz}$ are termed 'neurotonic'. ${ }^{[34]}$ Neurotonic activity results from intense nerve stimulation, nerve irritation or nerve damage. ${ }^{[34]}$

Romstöck et al..$^{[33]}$ identified three distinct types of train MUPs: A, B and C trains. The occurrence of A train - a sinusoidal, symmetrical sequence of high-frequency and low-amplitude (100-200 mV) signals, with a duration lasting from milliseconds to seconds and a short onset and offset correlated with additional post-operative facial nerve paresis. The $B$ and $C$ trains were found be to be irrelevant with respect to post-operative outcome.

As described by Romstöck et al. ${ }^{[33]} \mathrm{B}$ trains manifest either in a spike or burst pattern and are distinguished by their gradual onset, low amplitudes and average duration lasting minutes to hours. C trains, on the other hand, are irregular waveforms of varying amplitudes that bear resemblance to interference.

In addition, to detection of neuronal damage, EMG may be helpful in the delineation of the anatomical location of the facial nerve. ${ }^{[35]}$ Direct and definite visualisation of the nerve may not be always possible due to the tumour and its capsule altering the trajectory of the nerve. By determining the magnitude of the current required for muscle stimulation, the proximity of the nerve to the stimulating probe is estimated. When the facial nerve is very near to the stimulating probe and highly exposed to it with a little tumour, tissue or bone covering, very 


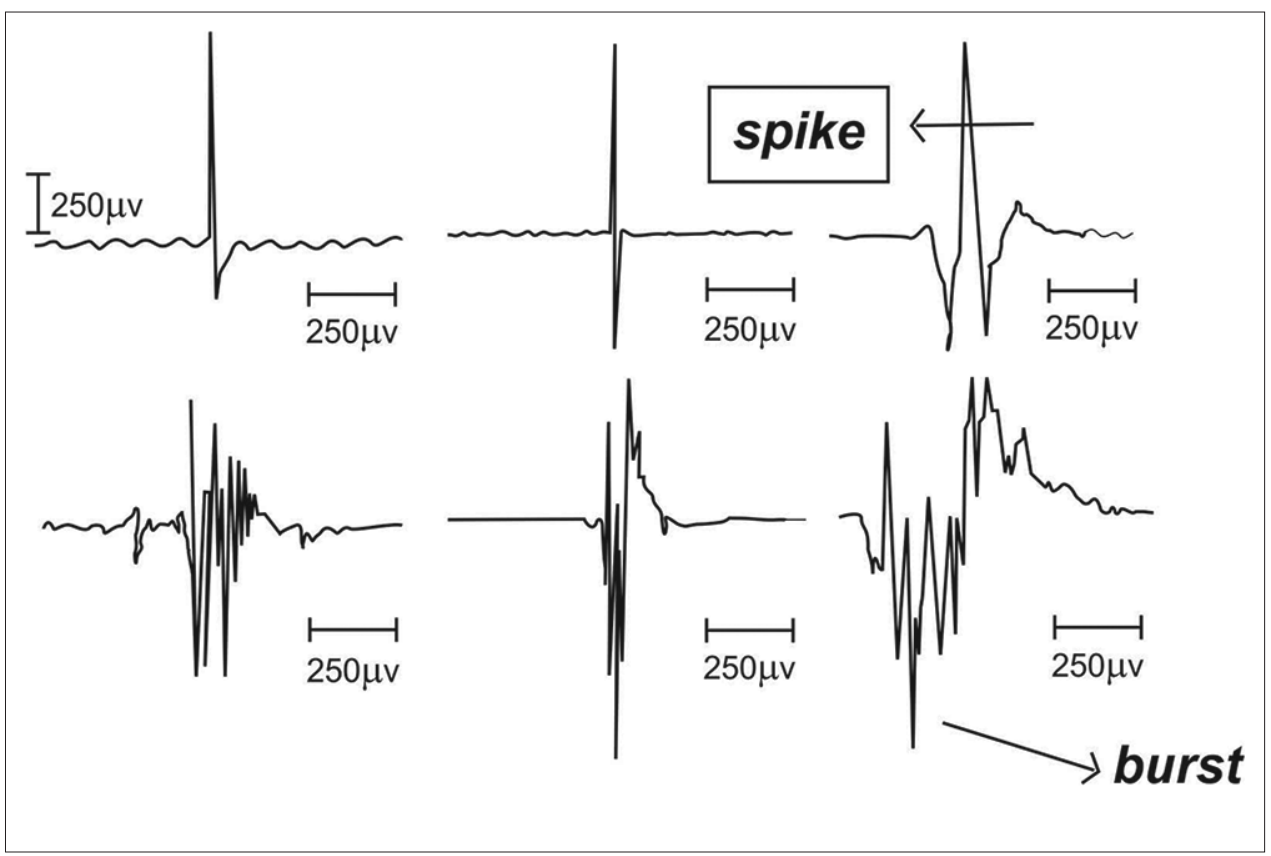

Figure 2: Electromyography motor unit potential morphologies demonstrating spikes (upper) and bursts (lower)

low currents around $0.2 \mathrm{~mA}$ stimulate it. ${ }^{[35]}$ The higher currents $>0.5 \mathrm{~mA}$ signifies the presence of a sizable tissue or bone barrier between the nerve from the probe. ${ }^{[36]}$

EMG may prevent the unplanned manipulation of the facial nerve by emitting a warning noise, whenever muscle stimulation is detected. Thus, the surgeon may stop the current actions or exercise extreme caution during dissection avoiding the damage to the nerve. ${ }^{[33]}$

EMG may help in the assessment of the residual function of the nerve post-operatively. The pre-operative and post-operative stimulation thresholds are compared. If a higher post-operative current is required, this may signify intra-operative nerve injury. ${ }^{[37]}$

It should be kept in mind that during resection there may be no suggestive electrophysiological changes of neural injury on facial nerve monitoring, but this may not always translate into the integrity of the facial nerve. ${ }^{[37]}$ Possible causes may be poor data input due to micro instruments used to cauterise the tissue or tumour surrounding the facial nerve. ${ }^{[34,38]}$

There is also a risk of EMG induced electrical injury by the stimulating probe. This may be minimised by application of pulsed stimulation ${ }^{[39]}$ and the use of monopolar rather than bipolar current. ${ }^{[40]}$

\section{DIRECT OBSERVATION/VIDEO MONITORING}

Intra-operative video monitoring (IOVM) may supplement the EMG by better visualisation of facial muscle contractions. During IOVM, an anaesthesia mask containing infrared cameras is fastened to the patient's face. These cameras record under the operative drapes. ${ }^{[38]}$ The camera view is magnified so that even minute movements may be detected by the naked eye. ${ }^{[38]}$ The images are projected on a four-way split screen: Two focus on movements of the facial muscles, another displays the microscopic operating field and the remaining screen projects the EMG tracings. ${ }^{[4]]}$

However, studies have shown that the use of EMG alone exhibited higher sensitivity in detecting facial nerve activation when compared with a combination of EMG with IOVM.

\section{Visual evoked potentials}

VEPs generated in the visual cortex are produced in response to light stimulation of the eyes. In awake patients for various diagnostic purposes the potentials are generated using alternately high-contrast checkerboard pattern. Under anaesthesia, flash stimulation is utilised through closed eyelids or through stimulators mounted on scleral caps. The VEPs that are recorded intra-operatively are generally recorded using electrodes placed on the scalp at $\mathrm{Cz}$ and $\mathrm{Oz}$ locations. VEPs are used for monitoring the anterior visual pathways during craniofacial procedures, pituitary surgery and surgery in the retrochiasmatic visual tracts. VEPs have limited practical utility as the large, bulky 'goggles' used for stimulation pose technical problems and the bilateral nature of the response makes interpretation difficult, and they are profoundly affected by the anaesthetic agents. 


\section{Motor evoked potentials}

SSEP monitoring was used in the past to reduce the risk of motor system injury. This was based on the assumption that the sensory and motor pathway are in proximity. Hence, pathophysiological changes affecting motor pathways may affect the sensory pathways causing changes in SSEP. Nuwer et al. found that SSEP monitoring could decrease the risk of paraplegia by $50 \%$ during scoliosis surgery. ${ }^{[42]}$ However, many cases of motor injury without SSEP warning occurred. ${ }^{[42]}$ This was due to the distinct anatomy and vascular supply to the sensory and motor systems. Thus, the rationale for MEP monitoring is to directly test the motor system during surgery.

\section{ANATOMY AND PHYSIOLOGY}

\section{Motor cortex}

Primary motor cortex which lies in the pre-central gyrus controls the voluntary movement of the body and its destruction may cause permanent weakness. It is organised with tongue and face motor neurons near the sylvian fissure, hand and arm neurons in its middle convexity, and leg and foot neurons from its crest to mesial parasagittal region. The volume of motor gyrus innervating distal limb, tongue and lower facial muscles is greater than other muscles. Hence, distal limb muscles are used primarily as the MEP recording sites.

\section{Corticospinal tract, corticobulbar tract and indirect motor pathways}

The corticospinal tract is the only direct descending connection between the cortex and spinal cord and is important for voluntary movement. Pure corticospinal tract lesions cause paralysis which partially resolves over time to impaired strength and impaired control of distal limb muscles with a positive Babinski sign. Nearly, $75-90 \%$ of corticospinal fibres cross the midline at the pyramidal decussation and then descend laterally, remaining uncrossed fibres descend ventrally. Decussation makes MEP thresholds lower and amplitudes larger contralateral to the stimulated hemisphere.

The corticobulbar tract which connects the cortex and brainstem motor nuclei is necessary for voluntary movements of the cranial muscles. Projections to most motor nuclei are bilateral. Hence, unilateral cortical stimulation tends to produce bilateral movements and unilateral lesions produce mild if any weakness. However, projections to lower face and tongue motor nuclei are mostly contralateral so that unilateral cortical stimulation produces contralateral movement and unilateral lesions produce contralateral weakness of these muscles.

The indirect motor pathways consist of cortico-brainstem-spinal relays. Their axons descend with corticospinal fibres to synapse in the brainstem. From there, rubrospinal, vestibulospinal, reticulospinal and tectospinal tracts travel down the ventral and lateral spinal cord white matter. Indirect motor pathways control the axial and integrated body-limb movements, posture and muscle tone. They probably do not directly contribute to MEPs, but might influence muscle MEPs through background synaptic facilitation. ${ }^{[43]}$

MEP monitoring for intra-operative monitoring requires transcranial stimulation of the motor cortex by electrical or magnetic means to produce a descending response that traverses the corticospinal tracts and eventually generates a measurable response.

\section{Recording of motor evoked potentials}

Tanscranial electrical stimulation (TcES) is the standard method used to generate an MEP response. TcES consists of usually 3-7 electrical pulses of 100-400 V (up to 1,000 V is possible) through electrodes most commonly placed a few centimetres anterior to the somatosensory electrodes at C3-C4 (International 10-20 system). The stimulus is most often $0.2 \mathrm{~ms}$ in duration but can be varied up to 0.5 $\mathrm{ms}$ and the interstimulus interval (the period between stimuli) varies between 2 and 4 ms. ${ }^{[35]}$ Cork screw scalp electrodes increase surface area and reduce the risk of burns from the high energy stimulus.

The applied current directly stimulates pyramidal cells of the motor cortex, resulting in a wave of depolarisation that often only involves $4-5 \%$ of the corticospinal tract. When this wave of depolarisation is measured by electrodes in the epidural space, it is termed the $\mathrm{D}$ (direct) wave. D-waves are compound corticospinal action potentials initiated by direct axonal activation and having approximately $50 \mathrm{~m} / \mathrm{s}$ conduction velocity. ${ }^{[4]}$ Additional trans-synaptic activation of inter-nuncial pathways in the cortex results in a series of smaller waves, called I (indirect) waves, which follow the D-wave. The motor pathway descends from the motor cortex, crossing the midline in the lower lateral brainstem and descending in the ipsilateral and anterior funiculi of the spinal cord. The electrical activity of the D- and I- waves summate in the anterior horn cell, resulting in activation of the peripheral nerve, which produces a CMAP.

\section{Interpretation criteria for the muscle motor evoked potentials}

The muscle MEP (CMAP) is the most commonly used TcMEP. Recordings are of high amplitude and can be obtained with a single trial. The problem with the muscle MEP is that the waveform is complex. Thus, many schemes have been devised to try to determine when there is a significant change. ${ }^{[45]}$ 


\section{Threshold criterion}

It was introduced by Calancie et al. ${ }^{[46]}$ and did not depend on amplitude. According to this criterion, a stimulus threshold for obtaining a muscle MEP increases when there is damage to the corticospinal tract. Typically, an increase of more than $100 \mathrm{~V}$ in the threshold required for eliciting responses that are persistent for 1 or more hours and not due to systemic factors were highly correlated with post-operative deficits. This criterion yielded a sensitivity and specificity of $100 \%$ for the post-operative deficit. The difficulty with this criterion is that threshold generally increases gradually during surgery and are significantly influenced by an even small change in anaesthesia. ${ }^{[47]}$

\section{The amplitude criterion}

No doubt it indicates a significant change, but is not always indicative of permanent injury. Sala et al. ${ }^{[48]}$ during intra-medullary tumour surgery reported a loss of MEP without more than a $50 \%$ of change in the D-wave was associated only with transient neurological deficits. However, in most spinal surgeries other than cord tumours wave recording is difficult and limited. ${ }^{[49]}$ Other authors ${ }^{[50]}$ have suggested a reduction in amplitude of $50 \%$ or more be taken as significant. The problem with this criterion is that there are some natural variations in the muscle MEP, thereby, giving false positive and false negative alarms. Although some investigators have proposed a change in pattern and duration of the MEP morphology from polyphasic to biphasic, ${ }^{[51]}$ it is still under investigations.

\section{All or nothing criterion}

This is the most widely cited and used criterion, given the inherent variability of signals in MEP monitoring. ${ }^{[52]}$ Based on this approach, a total disappearance of the MEP signal indicates a clinically significant event. Owing to all or none nature of this interpretation, it has been proposed that this method is not sensitive enough in detection of subtle deficits of the corticospinal tract that may still result in post-operative motor deficits and are potentially correctable if detected. ${ }^{[53]}$

A modification of the all-or-nothing criterion involves measuring the baseline CMAP amplitude, then measuring the relative change in amplitude to determine if a clinically significant change has occurred. The amplitude criterion, as described by Langeloo et al., ${ }^{[54]}$ uses an $80 \%$ amplitude loss in at least 1 out of 6 recording sites as a clinically significant change.

Each of these criteria is considered valid, and the specific criterion employed in a given case should be discussed in the pre-operative period between the operating team and monitoring team.
Muscle MEPs are considered the gold standard for detection of new post-operative motor deficits, with (sensitivity ranging from $75 \%$ to $100 \%$ and specificity ranging from $84 \%$ to $100 \% .{ }^{[55,56]}$

\section{APPLICATIONS OF MOTOR EVOKED POTENTIAL MONITORING IN NEUROSURGICAL PROCEDURES}

\section{Intra-medullary spinal cord tumour surgery}

Gross total tumour removal would be a curative goal for intra-medullary spinal cord tumour surgery. Dorsal myelotomy leads to SSEP loss. However, this should not stop the surgical procedure. ${ }^{[57]}$ Mild or temporary severe motor deficits may be acceptable for the patient but a permanent paraplegia is an excessive price for total tumour resection and its prevention provides strong motivation for monitoring.

It has been seen that when SSEP, muscle MEP and D-wave monitoring was done, there was an improved outcome. It was seen that muscle MEP disappearance modified surgery, but more than $50 \% \mathrm{D}$-wave amplitude decrement was the major indication to stop surgery. ${ }^{[48]}$

\section{Spinal deformity surgery}

The incidence of cord injury in scoliosis surgery is very low $(0.6 \%) .{ }^{[42]}$ With the introduction of SSEP monitoring risk of paraplegia has been reduced. ${ }^{[42]}$ However, selective injury of the unassessed motor system still occurs without SSEP warning. The MEP monitoring should be helpful in improving the outcome. Studies have shown that muscle MEP monitoring may further reduce paraplegia risk. ${ }^{[54,58]}$

\section{Posterior fossa tumour surgery}

Muscle MEPs may enhance brainstem and $\mathrm{CN}$ protection during surgery for tumours in the posterior fossa. Applying C3-Cz and C4-Cz TcES to evoke right and left facial and hand muscle responses provides an assessment of bilateral corticospinal and corticobulbar tract as well as facial nerve assessment.

\section{Intra-cranial aneurysm surgery}

Changes in the evoked potentials are helpful in detection of the early ischaemia. If prompt measures are instituted, this helps in preventing the development of infarction. Median and tibial nerve SSEP monitoring is done for the detection of cortical ischaemia in the distribution of the MCA's posterior division and the distal anterior cerebral artery. Cortical ischaemia in other vascular territories might go undetected. Szelényi et al. reported a patient with infarction in the territory of the anterior division of the MCA and consequent hemiplegia who did not exhibit intra-operative median SSEP alteration 
or sensory deficit. ${ }^{[59]}$ Similarly, infarction of other deep structures including motor pathways can go undetected. ${ }^{[6,66]}$ Consequently, MEP monitoring has been introduced to evaluate motor cortex and corticospinal pathways descending through the corona radiata, internal capsule, cerebral peduncle, basis pontis and pyramids. Muscle MEPs have been shown to detect motor pathway ischaemia or infarction undetected by SEPs, and to provide greater sensitivity and earlier warning. ${ }^{[0,61]}$ Several patients have had MEP loss reversed after intervention and it seems likely that an overall improvement in patient outcome occurs. ${ }^{[33]}$

\section{Peri-rolandic brain surgery}

Electrically evoked motor activity for peri-rolandic brain surgery involves classical mapping with $50-60 \mathrm{~Hz}, 1-5 \mathrm{~s}$ pulse trains while observing the conscious patient for movement. During general anaesthesia, higher stimulus intensities are needed and the success rate diminishes. Various studies suggest that D-wave monitoring is helpful as D-wave preservation indicates primary motor cortex and corticospinal tract integrity ${ }^{[62,63]}$

\section{Limitations of motor evoked potentials}

- Extreme sensitivity to inhalational anaesthetics

- They cannot be monitored continuously.

The effect of pre-operative damage to the motor pathways and the Tc motor evoked potentials

One needs to understand that the condition of motor pathways prior to surgery is very crucial to the generation of the CMAP. In the presence of pre-operative injury, even if the patient has good power pre-operatively, the MEPs may be difficult to record. This is because activation of anterior horn cell requires a highly synchronised volley of inputs that can easily be desynchronised by a minor disruption of conduction. ${ }^{[45]}$

\section{Direct waves (D waves)}

These waves are generated through transcranial stimulation and monitored directly at the spinal cord level through the placement of epidural recording electrode caudal to the region at risk. In contrast to CMAP monitoring, D-waves are relatively resistant to anaesthetic effects and allow the use of muscle relaxants for paralysing the patient, thereby, eliminating risks associated with patient movement. ${ }^{[49,64]}$

In general, a $20 \%$ decrease in D-wave amplitude is considered to be a preliminary warning, whereas a $50 \%$ reduction in amplitude is indicates a significant neurological injury.

The predominant clinical application of D-waves lies in intra-medullary spinal cord tumour resection. Kothbauer et al. ${ }^{[52]}$ initially reported that D-waves were superior to CMAPs in predicting long-term motor status following intra-medullary spinal cord tumour removal. Specifically, they observed that patients with loss of CMAPs but preserved D-waves tended to have a transient post-operative weakness that resolved by 1-2 months follow-up. In contrast, the patients with loss of CMAPs as well as D-waves decrements more than $50 \%$ were more likely to have a lasting deficit. Thus, use of D-waves permitted for safer, more aggressive resection than would otherwise have been possible. Sala et al. ${ }^{[48]}$ too, reported similar observation. D-waves are currently believed to be the gold standard for motor pathways monitoring during intra-medullary spinal cord tumour resection. ${ }^{[64]}$

D-waves are not frequently monitored during deformity surgery owing to $27 \%$ of false positive incidence which according to Ulkatan et al.$^{[49]}$ is as a result of rotation of the corticospinal tract relative to recording electrode during spinal curvature correction. Furthermore, the lack of nerve root and cauda equina monitoring with D-waves may limit their use in these patients.

These waves do not delineate laterality:

- D-waves give a high false positive rate.${ }^{[49]}$ Monitoring of scoliosis surgery with epidurally recorded MEPs (D-Waves) revealed false results ${ }^{[49]}$

- Warning of vascular cord comprises is delayed when monitoring D-waves compared with CMAPs monitoring.

\section{Complications}

Potentially following complications can result from TcMEPs.

\section{Electrochemical injury}

The 3-7 pulse direct cortical trains used for muscle MEP monitoring are monophasic. Their extreme brevity with the better capacitive coupling limits faradic current. There have been no reported clinical signs of toxicity so far, although the histologic confirmation is lacking. Currently, it appears to be safe to apply this technique. ${ }^{[43]}$

\section{Excitotoxicity}

Animal experiments have shown that chronic direct cortical stimulation with $50 \mathrm{~Hz}$ biphasic pulse trains lasting many hours may lead to excitotoxic neuronal damage. ${ }^{[65]}$

However, in humans Gordon et al. found no histological damage after $50-\mathrm{Hz}$ intermittent biphasic pulse trains lasting up to $5 \mathrm{~s}{ }^{[66]}$ Hence, in the clinical scenario, cerebral excitotoxicity appears to be unlikely. There are no reports of clinical symptoms or signs suggesting such an injury. However, histologic confirmation is lacking and it is better to avoid needlessly-high intensity currents. 


\section{Seizures}

It has been seen that $50-60 \mathrm{~Hz}$ direct cortical pulse-trains lasting 1-5 s frequently induce after discharges (seizure patterns) that build to clinical seizures in $5-20 \%$ of patients. ${ }^{[67]}$ Most of these seizures are self-limited or readily aborted with a variety of techniques. ${ }^{[68]}$ Interestingly, to note that these after discharges can be terminated by the application of a brief burst of cortical electrical stimulation. ${ }^{[69]}$ Fortunately, the incidence of these seizures is very rare without morbidity. ${ }^{[65]}$

\section{Cardiovascular alterations}

Cardiac arrhythmia or blood pressure alteration may occur with pulse-train. Transcranial electrical stimulation due to deep current penetration to the hypothalamus or brainstem is a possible cause. ${ }^{[65]}$

Other reported complications are tongue laceration, scalp burn at the site of stimulating electrodes, jaw fracture and awareness. ${ }^{[70]}$ It is prudent to avoid cork screw electrode in the presence of open fontanelle.

\section{Limitations of motor evoked potentials}

They cannot be recorded continuously throughout the surgery. This represents a serious drawback of muscle MEPs as this may cause a delay in recognition of neurological injury. The protocol of stimulation should be discussed with the surgeon in the pre-operative period. The protocol ranges from only running MEPs at surgeon's request, to periodic monitoring at a set interval of time.

Since muscle relaxants are avoided during MEP monitoring, this introduces the risk of patient movement during stimulation which may cause serious neurological injury if the surgeon is not informed immediately before the stimulation.

\section{ANAESTHETIC CONSIDERATIONS DURING EVOKED POTENTIAL MONITORING}

\section{Effects of anaesthetics on somatosensory evoked potentials}

As anaesthetic agents reduce the synaptic transmission, their effect will be on the first synapse in the neural pathway at the nucleus cuneatus in the brainstem. Hence, the cortical responses will be affected markedly. The effect on those responses which are recorded at the cervical spine and the more peripheral sites (epidural space and peripheral nerves) is minimal.

Anaesthetic agents produce gating of sensory information at the thalamus. This leads to a dependent reduction seen by the inhalational agents. There is a dramatic decrease in the midlatency auditory evoked response, ${ }^{[71]}$ as the concentration of the inhalational agent increases.
Volatile anaesthetics produce an increase in latency and a decrease in amplitude of cortical sensory responses until they cannot reliably be detected (at about 1-1.5 minimal alveolar concentration).

\section{Effects on motor evoked potentials}

The genesis of the D-wave in the epidural space does not involve synaptic transmission; hence, they are little affected by the anaesthetics. As the I-waves are generated by synaptic mechanisms, they get progressively reduced as the dose of the anaesthetic agents increases ${ }^{[72]} \mathrm{A}$ stage may reach when the loss of I-waves, does not result in the activation of the anterior horn cell and the production of a CMAP is blocked. ${ }^{[73]}$

Nitrous oxide $\left(\mathrm{N}_{2} \mathrm{O}\right)$ produces amplitude reduction and latency increases in cortical sensory responses or MEP CMAP responses when used alone or when combined with the halogenated inhalational agent. The effects of $\mathrm{N}_{2} \mathrm{O}$ on subcortical, epidural and peripheral nerve responses are minimal.

Opioids alter the SSEPs minimally when compared with inhalation agents. They have become a popular component of total intravenous anaesthesia (TIVA). They cause little changes in spinal and subcortical SSEP recording. There is a slight reduction in cortical amplitude and increase of latency in the late cortical responses (latency more than $100 \mathrm{~ms}$ ). ${ }^{[74]}$

Ketamine has been found to enhance the cortical SSEP amplitude. Because of these properties, ketamine is a useful agent for eliciting responses that are usually difficult to record under anaesthesia. Its maximum effect on amplitude is observed within 2-10 min after bolus administration. ${ }^{[74]}$

Thiopentone: Immediately the following induction with thiopentone, there is a transient decrease in amplitude and increase in latency of cortical sensory responses. The effect lasts $<10 \mathrm{~min} \cdot{ }^{[75]}$

Propofol is most commonly used sedative component of TIVA when SSEPs and MEPs are monitored. Induction produces amplitude depression in cortical SSEPs, with rapid recovery after termination of infusion. ${ }^{[7]}$ Recordings in the epidural space are unaffected, consistent with the site of anaesthetic action of propofol in the cerebral cortex. The rapid metabolism of propofol makes it an excellent drug as its sedative effect and its related effects on evoked responses can be adjusted quickly.

An extensive critical appraisal of anaesthetic considerations for evoked potentials monitoring has been published in a previous issue of this journal. ${ }^{[7]]}$ The reader is suggested to go through it for a detailed review on the subject. 


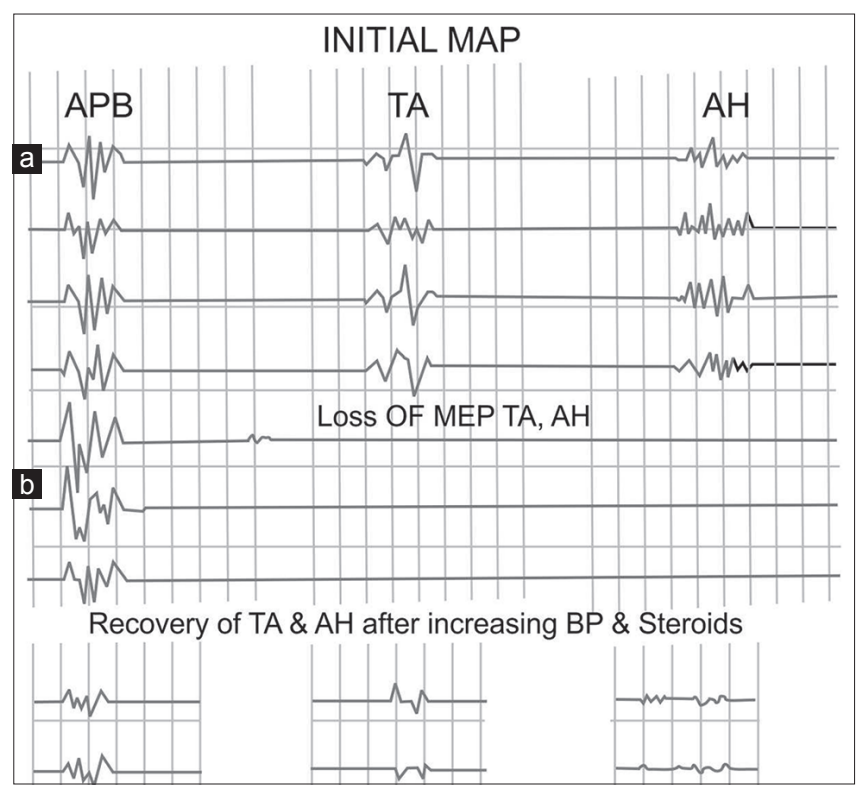

Figure 3: Recovery of motor evoked potential response after an intra-operative loss. During a posterior cervical fusion of C5 to T4 the patient abruptly lost motor evoked potential responses in both lower extremities (14:13:15). After blood pressure elevation and steroid administration, motor evoked potential response returned only on the left (18:35:53). The patient had weakness on the left, which resolved over 2 weeks. On the right, patient had a dense hemiparesis that had not changed 3 months after surgery

\section{What to do when neurophysiological signal changes?}

The first step in such situation is to check for unintentional lead disconnection. The surgeon should release any distraction on the cord and/or remove pedicle screw, hook cable, corrective rod, etc., Rule out hypothermia and severe anaemia. Next evaluate anaesthesia technique to rule out any recent change in the technique or inadvertent administration of a hypnotic agent or sudden increase of anaesthetic depth. Then increase mean arterial pressure to at least $80 \mathrm{mmHg}$. The surgeon should ensure that there is no inadvertent mass effect on the cord [Figure 3]. Following the above steps recheck signals, and if still reduced/absent perform wake up test. If extremities show no movement with wake up a test or if the test is not feasible, then consider the removal of any implant placed immediately prior to signal change. The presence of neurological deficit upon awakening warrants further radiological evaluation. Persistent monitoring alert or deficit detected with wake up test requires a procedure to be staged.

The summary of important intra-operative neurophysiological monitoring-related questions to include in the pre-operative period: ${ }^{[27]}$

- What monitoring modalities are most appropriate for the case at hand? What types of neurological deficits are most likely?

- What anaesthesia protocol will optimise the acquisition of neurophysiological signals? Is TIVA indicated? Can muscle relaxant be used?
- What alarm criteria will be used for each monitoring modality?

- What actions will be taken in the setting of a positive signal?

- Are new techniques involved? How will they be implemented?

\section{CONCLUSION}

The IONM has become mandatory in the European and American spinal and neurosurgical units. The current body of evidence shows that IONM is effective in predicting an increased risk of the adverse outcomes. ${ }^{[76]}$ According to an international survey involving more than 100 neurosurgeons from 16 countries on the availability and importance of IONM, neurosurgeons with IONM experience of more than 5 years stated IONM had less influence on the course of their surgeries than did surgeons with less experience with tool. ${ }^{[77]}$ IONM helped budding neurosurgeon learn which step of a surgery caused an injury, and helped them refine their surgical skills.

\section{Financial support and sponsorship Nil.}

\section{Conflicts of interest}

There are no conflicts of interest.

\section{REFERENCES}

1. Aminoff MJ, Eisen AA. AAEM minimonograph 19: Somatosensory evoked potentials. Muscle Nerve 1998;21:277-90.

2. Hume AL, Cant BR. Conduction time in central somatosensory pathways in man. Electroencephalogr Clin Neurophysiol 1978;45:361-75.

3. Branston NM, Symon L, Crockard HA, Pasztor E. Relationship between the cortical evoked potential and local cortical blood flow following acute middle cerebral artery occlusion in the baboon. Exp Neurol 1974;45:195-208.

4. Astrup J, Symon L, Branston NM, Lassen NA. Cortical evoked potential and extracellular $\mathrm{K}+$ and $\mathrm{H}+$ at critical levels of brain ischemia. Stroke 1977;8:51-7.

5. Symon L, Hargadine J, Zawirski M, Branston N. Central conduction time as an index of ischaemia in subarachnoid haemorrhage. J Neurol Sci 1979;44:95-103.

6. Momma F, Wang AD, Symon L. Effects of temporary arterial occlusion on somatosensory evoked responses in aneurysm surgery. Surg Neurol 1987;27:343-52.

7. Symon L, Murota T. Intraoperative monitoring of somatosensory evoked potentials during intracranial vascular surgery. In: Desmedt JE, editor. Neuromonitoring in Surgery. Amsterdam: Elsevier Science; 1989. p. 263-74.

8. Guo L, Gelb AW. The use of motor evoked potential monitoring during cerebral aneurysm surgery to predict pure motor deficits due to subcortical ischemia. Clin Neurophysiol 2011;122:648-55.

9. Møller AR. Intraoperative Neurophysiological Monitoring. $2^{\text {nd }}$ ed. Dallas, TX: Humana Press Inc.; c2006. p. 126.

10. Klem GH, Liiders HO, Jasper HH, Elger C. The ten-twenty electrode system of the International Federation. The 
International Federation of Clinical Neurophysiology. Electroencephalogr Clin Neurophysiol Suppl 1999;52:3-6.

11. Desmedt JE, Cheron G. Non-cephalic reference recording of early somatosensory potentials to finger stimulation in adult or aging normal man: Differentiation of widespread $\mathrm{N} 18$ and contralateral N20 from the prerolandic P22 and N30 components. Electroencephalogr Clin Neurophysiol 1981;52:553-70.

12. Hilibrand AS, Schwartz DM, Sethuraman V, Vaccaro AR, Albert TJ. Comparison of transcranial electric motor and somatosensory evoked potential monitoring during cervical spine surgery. J Bone Joint Surg Am 2004;86-A: 1248-53.

13. Youssef AS, Downes AE. Intraoperative neurophysiological monitoring in vestibular schwannoma surgery: Advances and clinical implications. Neurosurg Focus 2009;27:E9.

14. Petrova LD. Brainstem auditory evoked potentials. Am J Electroneurodiagnostic Technol 2009;49:317-32.

15. Simon MV. Neurophysiologic intraoperative monitoring of the vestibulocochlear nerve. J Clin Neurophysiol 2011;28:566-81.

16. Polo G, Fischer C, Sindou MP, Marneffe V. Brainstem auditory evoked potential monitoring during microvascular decompression for hemifacial spasm: Intraoperative brainstem auditory evoked potential changes and warning values to prevent hearing loss - Prospective study in a consecutive series of 84 patients. Neurosurgery 2004;54:97-104.

17. Markand ON. Brainstem auditory evoked potentials. J Clin Neurophysiol 1994;11:319-42.

18. Phillips DJ, Kobylarz EJ, De Peralta ET, Stieg PE, Selesnick SH. Predictive factors of hearing preservation after surgical resection of small vestibular schwannomas. Otol Neurotol 2010;31:1463-8.

19. Colletti V, Fiorino FG, Mocella S, Policante Z. ECochG, CNAP and $A B R$ monitoring during vestibular schwannoma surgery. Audiology 1998;37:27-37.

20. Yamakami I, Oka N, Yamaura A. Intraoperative monitoring of cochlear nerve compound action potential in cerebellopontine angle tumour removal. J Clin Neurosci 2003;10:567-70.

21. Yamakami I, Yoshinori H, Saeki N, Wada M, Oka N. Hearing preservation and intraoperative auditory brainstem response and cochlear nerve compound action potential monitoring in the removal of small acoustic neurinoma via the retrosigmoid approach. J Neurol Neurosurg Psychiatry 2009;80:218-27.

22. Arts HA, Telian SA, El-Kashlan H, Thompson BG. Hearing preservation and facial nerve outcomes in vestibular schwannoma surgery: Results using the middle cranial fossa approach. Otol Neurotol 2006;27:234-41.

23. Bose B, Sestokas AK, Schwartz DM. Neurophysiological detection of iatrogenic C-5 nerve deficit during anterior cervical spinal surgery. J Neurosurg Spine 2007;6:381-5.

24. Jimenez JC, Sani S, Braverman B, Deutsch H, Ratliff JK. Palsies of the fifth cervical nerve root after cervical decompression: Prevention using continuous intraoperative electromyography monitoring. J Neurosurg Spine 2005;3:92-7.

25. Obi T, Mochizuki M, Isobe K, Mizoguchi K, Takatsu M, Nishimura Y. Mechanically elicited nerve root discharge: Mechanical irritation and waveform. Acta Neurol Scand 1999;100:185-8.

26. Quraishi NA, Lewis SJ, Kelleher MO, Sarjeant R, Rampersaud YR, Fehlings MG. Intraoperative multimodality monitoring in adult spinal deformity: Analysis of a prospective series of one hundred two cases with independent evaluation. Spine (Phila Pa 1976) 2009;34:1504-12.

27. Lall RR, Lall RR, Hauptman JS, Munoz C, Cybulski GR, Koski T, et al. Intraoperative neurophysiological monitoring in spine surgery: Indications, efficacy, and role of the preoperative checklist. Neurosurg Focus 2012;33:E10.

28. Raynor BL, Lenke LG, Bridwell KH, Taylor BA, Padberg AM.
Correlation between low triggered electromyographic thresholds and lumbar pedicle screw malposition: Analysis of 4857 screws. Spine (Phila Pa 1976) 2007;32:2673-8.

29. Parker SL, Amin AG, Farber SH, McGirt MJ, Sciubba DM, Wolinsky JP, et al. Ability of electromyographic monitoring to determine the presence of malpositioned pedicle screws in the lumbosacral spine: Analysis of 2450 consecutively placed screws. J Neurosurg Spine 2011;15:130-5.

30. Raynor BL, Lenke LG, Kim Y, Hanson DS, Wilson-Holden TJ, Bridwell $\mathrm{KH}$, et al. Can triggered electromyograph thresholds predict safe thoracic pedicle screw placement? Spine (Phila Pa 1976) 2002;27:2030-5.

31. Shi YB, Binette M, Martin WH, Pearson JM, Hart RA. Electrical stimulation for intraoperative evaluation of thoracic pedicle screw placement. Spine (Phila Pa 1976) 2003;28:595-601.

32. Grayeli AB, Guindi S, Kalamarides M, El Garem H, Smail M, Rey A, et al. Four-channel electromyography of the facial nerve in vestibular schwannoma surgery: Sensitivity and prognostic value for short-term facial function outcome. Otol Neurotol 2005;26:114-20.

33. Romstöck J, Strauss C, Fahlbusch R. Continuous electromyography monitoring of motor cranial nerves during cerebellopontine angle surgery. J Neurosurg 2000;93:586-93.

34. Harper CM. Intraoperative cranial nerve monitoring. Muscle Nerve 2004;29:339-51.

35. Delgado TE, Bucheit WA, Rosenholtz HR, Chrissian S. Intraoperative monitoring of facila muscle evoked responses obtained by intracranial stimulation of the facila nerve: A more accurate technique for facila nerve dissection. Neurosurgery 1979;4:418-21.

36. Silverstein H, Smouha EE, Jones R. Routine intraoperative facial nerve monitoring during otologic surgery. Am J Otol 1988;9:269-75.

37. Marin P, Pouliot D, Fradet G. Facial nerve outcome with a peroperative stimulation threshold under $0.05 \mathrm{~mA}$. Laryngoscope 2011;121:2295-8.

38. Murphy EK. Use of an infrared camera to improve the outcome of facial nerve monitoring. Am J Electroneurodiagnostic Technol 2008;48:38-47.

39. Eisele DW, Wang SJ, Orloff LA. Electrophysiologic facial nerve monitoring during parotidectomy. Head Neck 2010;32:399-405.

40. Møller AR, Jannetta PJ. Preservation of facial function during removal of acoustic neuromas. Use of monopolar constant-voltage stimulation and EMG. J Neurosurg 1984;61:757-60.

41. Filipo R, Pichi B, Bertoli GA, De Seta E. Video-based system for intraoperative facial nerve monitoring: Comparison with electromyography. Otol Neurotol 2002;23:594-7.

42. Nuwer MR, Dawson EG, Carlson LG, Kanim LE, Sherman JE. Somatosensory evoked potential spinal cord monitoring reduces neurologic deficits after scoliosis surgery: Results of a large multicenter survey. Electroencephalogr Clin Neurophysiol 1995;96:6-11.

43. Macdonald DB. Intraoperative motor evoked potential monitoring: Overview and update. J Clin Monit Comput 2006;20:347-77.

44. Amassian VE. Animal and human motor system neurophysiology related to intraoperative monitoring. In: Deletis V, Shils JL, editors. Neurophysiology in Neurosurgery. San Diego: Academic Press; 2002. p. 3-23.

45. Stecker MM. A review of intraoperative monitoring for spinal surgery. Surg Neurol Int 2012;3 Suppl 3:S174-87.

46. Calancie B, Harris W, Broton JG, Alexeeva $\mathrm{N}$ Green BA. "Threshold-level" multipulse transcranial electrical stimulation of motor cortex for intraoperative monitoring of spinal motor tracts: Description of method and comparison 
to somatosensory evoked potential monitoring. J Neurosurg 1998;88:457-70.

47. Langeloo DD, Journée HL, de Kleuver M, Grotenhuis JA. Criteria for transcranial electrical motor evoked potential monitoring during spinal deformity surgery A review and discussion of the literature. Neurophysiol Clin 2007;37:431-9.

48. Sala F, Palandri G, Basso E, Lanteri P, Deletis V, Faccioli F, et al. Motor evoked potential monitoring improves outcome after surgery for intramedullary spinal cord tumors: A historical control study. Neurosurgery 2006;58:1129-43.

49. Ulkatan S, Neuwirth M, Bitan F, Minardi C, Kokoszka A, Deletis V. Monitoring of scoliosis surgery with epidurally recorded motor evoked potentials (D wave) revealed false results. Clin Neurophysiol 2006;117:2093-101.

50. Krammer MJ, Wolf S, Schul DB, Gerstner W, Lumenta CB. Significance of intraoperative motor function monitoring using transcranial electrical motor evoked potentials (MEP) in patients with spinal and cranial lesions near the motor pathways. Br J Neurosurg 2009;23:48-55.

51. Quiñones-Hinojosa A, Lyon R, Zada G, Lamborn KR, Gupta N, Parsa AT, et al. Changes in transcranial motor evoked potentials during intramedullary spinal cord tumor resection correlate with postoperative motor function. Neurosurgery 2005;56:982-93.

52. Kothbauer KF, Deletis V, Epstein FJ. Motor-evoked potential monitoring for intramedullary spinal cord tumor surgery: Correlation of clinical and neurophysiological data in a series of 100 consecutive procedures. Neurosurg Focus 1998;4:e1.

53. Calancie B, Molano MR. Alarm criteria for motor-evoked potentials: What's wrong with the "presence-or-absence" approach? Spine (Phila Pa 1976) 2008;33:406-14.

54. Langeloo DD, Lelivelt A, Louis Journée $H$, Slappendel $R$, de Kleuver M. Transcranial electrical motor-evoked potential monitoring during surgery for spinal deformity: A study of 145 patients. Spine (Phila Pa 1976) 2003;28:1043-50.

55. Schwartz DM, Auerbach JD, Dormans JP, Flynn J, Drummond DS, Bowe JA, et al. Neurophysiological detection of impending spinal cord injury during scoliosis surgery. J Bone Joint Surg Am 2007;89:2440-9.

56. Hsu B, Cree AK, Lagopoulos J, Cummine JL. Transcranial motor-evoked potentials combined with response recording through compound muscle action potential as the sole modality of spinal cord monitoring in spinal deformity surgery. Spine (Phila Pa 1976) 2008;33:1100-6.

57. Kothbauer KF. Motor evoked potential monitoring for intramedullary spinal cord tumor surgery. In: Deletis V, Shils JL, editors. Neurophysiology in Neurosurgery. California: Academic Press; 2002. p. 73-92.

58. MacDonald DB, Al Zayed Z, Khoudeir I, Stigsby B. Monitoring scoliosis surgery with combined multiple pulse transcranial electric motor and cortical somatosensory-evoked potentials from the lower and upper extremities. Spine (Phila Pa 1976) 2003;28:194-203.

59. Szelényi A, Bueno de Camargo A, Flamm E, Deletis V. Neurophysiological criteria for intraoperative prediction of pure motor hemiplegia during aneurysm surgery. Case report. J Neurosurg 2003;99:575-8.

60. Sakuma J, Suzuki K, Sasaki T, Matsumoto M, Oinuma M, Kawakami M, et al. Monitoring and preventing blood flow insufficiency due to clip rotation after the treatment of internal carotid artery aneurysms. J Neurosurg 2004;100:960-2.

61. Horiuchi K, Suzuki K, Sasaki T, Matsumoto M, Sakuma J,
Konno Y, et al. Intraoperative monitoring of blood flow insufficiency during surgery of middle cerebral artery aneurysms. J Neurosurg 2005;103:275-83.

62. Yamamoto T, Katayama Y, Nagaoka T, Kobayashi K, Fukaya C. Intraoperative monitoring of the corticospinal motor evoked potential (D-wave): Clinical index for postoperative motor function and functional recovery. Neurol Med Chir (Tokyo) 2004;44:170-80.

63. Fujiki M, Furukawa Y, Kamida T, Anan M, Inoue R, Abe T, et al. Intraoperative corticomuscular motor evoked potentials for evaluation of motor function: A comparison with corticospinal D and I waves. J Neurosurg 2006;104:85-92.

64. Deletis V, Sala F. Intraoperative neurophysiological monitoring of the spinal cord during spinal cord and spine surgery: A review focus on the corticospinal tracts. Clin Neurophysiol 2008;119:248-64.

65. MacDonald DB. Safety of intraoperative transcranial electrical stimulation motor evoked potential monitoring. J Clin Neurophysiol 2002;19:416-29.

66. Gordon B, Lesser RP, Rance NE, Hart J Jr, Webber R, Uematsu $S$, et al. Parameters for direct cortical electrical stimulation in the human: Histopathologic confirmation. Electroencephalogr Clin Neurophysiol 1990;75:371-7.

67. Sartorius CJ, Wright G. Intraoperative brain mapping in a community setting - Technical considerations. Surg Neurol 1997;47:380-8.

68. Sartorius CJ, Berger MS. Rapid termination of intraoperative stimulation-evoked seizures with application of cold Ringer's lactate to the cortex. Technical note. J Neurosurg 1998;88:349-51.

69. Lesser RP, Kim SH, Beyderman L, Miglioretti DL, Webber WR, Bare $\mathrm{M}$, et al. Brief bursts of pulse stimulation terminate afterdischarges caused by cortical stimulation. Neurology 1999;53:2073-81.

70. Legatt AD. Current practice of motor evoked potential monitoring: Results of a survey. J Clin Neurophysiol 2002;19:454-60.

71. Sloan TB. Evoked potentials. In: Albin MA, editor. Textbook of Neuroanesthesia with Neurosurgical and Neuroscience Perspectives. New York: McGraw-Hill; 1997. p. 221.

72. Hicks RG, Woodforth IJ, Crawford MR, Stephen JP, Burke DJ. Some effects of isoflurane on I waves of the motor evoked potential. Br J Anaesth 1992;69:130-6.

73. Ubags LH, Kalkman CJ, Been HD. Influence of isoflurane on myogenic motor evoked potentials to single and multiple transcranial stimuli during nitrous oxide/opioid anesthesia. Neurosurgery 1998;43:90-4.

74. Bithal PK. Anaesthetic considerations for evoked potentials monitoring. J Neuroanaesth Crit Care 2014;1:2-12.

75. Koht A, Schütz W, Schmidt G, Schramm J, Watanabe E. Effects of etomidate, midazolam, and thiopental on median nerve somatosensory evoked potentials and the additive effects of fentanyl and nitrous oxide. Anesth Analg 1988;67:435-41.

76. Nuwer MR, Emerson RG, Galloway G, Legatt AD, Lopez J, Minahan R, et al. Evidence-based guideline update: Intraoperative spinal monitoring with somatosensory and transcranial electrical motor evoked potentials. J Clin Neurophysiol 2012;29:101-8.

77. Cabraja M, Stockhammer F, Mularski S, Suess O, Kombos T, Vajkoczy P. Neurophysiological intraoperative monitoring in neurosurgery: Aid or handicap? An international survey. Neurosurg Focus 2009;27:E2. 